\title{
Four-probe sensing of temperature during Joule heating of silicon
}

Pedersen, Asger Kjærgård; Madhi, Daena; Mirosnikov, Daniil; Jepsen, Peter Uhd; Petersen, Dirch Hjorth

Published in:

Review of Scientific Instruments

Link to article, DOI:

$10.1063 / 5.0033465$

Publication date:

2021

Document Version

Publisher's PDF, also known as Version of record

Link back to DTU Orbit

Citation (APA):

Pedersen, A. K., Madhi, D., Mirosnikov, D., Jepsen, P. U., \& Petersen, D. H. (2021). Four-probe sensing of temperature during Joule heating of silicon. Review of Scientific Instruments, 92(1), [014903].

https://doi.org/10.1063/5.0033465

\section{General rights}

Copyright and moral rights for the publications made accessible in the public portal are retained by the authors and/or other copyright owners and it is a condition of accessing publications that users recognise and abide by the legal requirements associated with these rights.

- Users may download and print one copy of any publication from the public portal for the purpose of private study or research.

- You may not further distribute the material or use it for any profit-making activity or commercial gain

- You may freely distribute the URL identifying the publication in the public portal 


\section{Four-probe sensing of temperature during Joule heating of silicon}

Cite as: Rev. Sci. Instrum. 92, 014903 (2021); https://doi.org/10.1063/5.0033465

Submitted: 21 October 2020 . Accepted: 30 December 2020 . Published Online: 26 January 2021

(D) Asger Kjærgård Pedersen, Daena Madhi, Daniil Mirosnikov, (D) Peter Uhd Jepsen, and (D) Dirch Hjorth Petersen

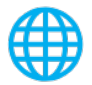

\section{ARTICLES YOU MAY BE INTERESTED IN}

Observation of photon antibunching with only one standard single-photon detector Review of Scientific Instruments 92, 013105 (2021); https://doi.org/10.1063/5.0038035

\section{Multiplexed lock-in thermography}

Review of Scientific Instruments 92, 014902 (2021); https://doi.org/10.1063/5.0029001

Development of in situ optical spectroscopy with high temporal resolution in an aberrationcorrected transmission electron microscope

Review of Scientific Instruments 92, 013704 (2021); https://doi.org/10.1063/5.0031115

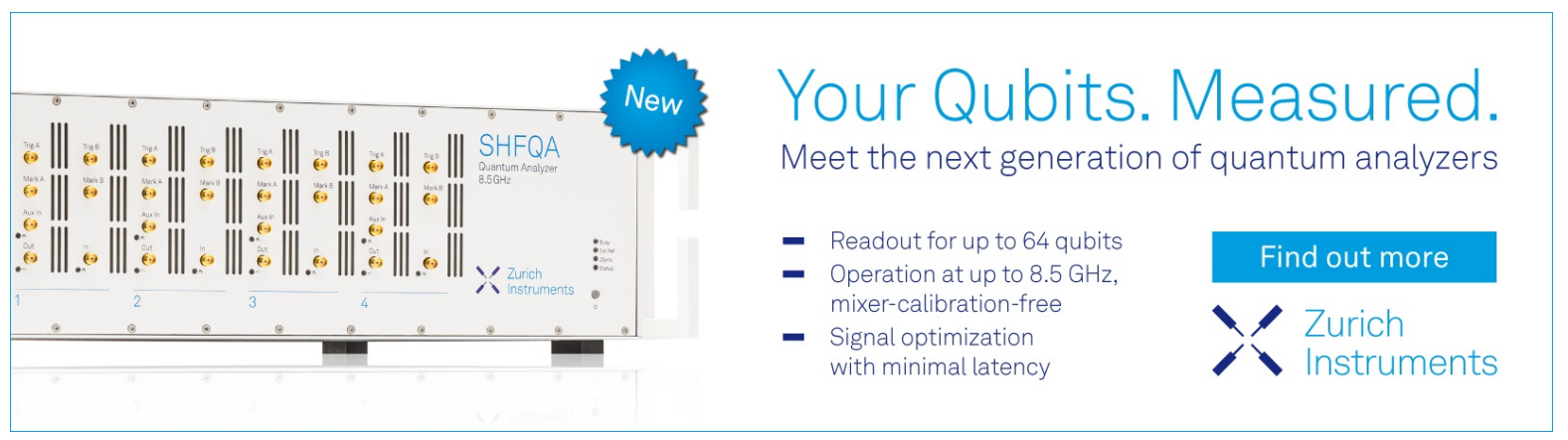




\title{
Four-probe sensing of temperature during Joule heating of silicon
}

\author{
Cite as: Rev. Sci. Instrum. 92, 014903 (2021); doi: 10.1063/5.0033465 \\ Submitted: 21 October 2020 - Accepted: 30 December 2020 • \\ Published Online: 26 January 2021
}

\author{
Asger Kjærgård Pedersen, ${ }^{1,}$ \\ and Dirch Hjorth Petersen \\ Daena Madhi, ${ }^{2}$ Daniil Mirosnikov, ${ }^{3}$ Peter Uhd Jepsen, ${ }^{2}$
}

\begin{abstract}
AFFILIATIONS
${ }^{1}$ DTU Energy-Department of Energy Conversion and Storage, Technical University of Denmark, DK-2800 Kongens Lyngby, Denmark

${ }^{2}$ DTU Fotonik-Department of Photonics Engineering, Technical University of Denmark, DK-2800 Kongens Lyngby, Denmark

${ }^{3}$ Topsil GlobalWafers A/S, DK-3600 Frederikssund, Denmark
\end{abstract}

a)asger1207@gmail.com

b) Author to whom correspondence should be addressed: dhpe@dtu.dk

\begin{abstract}
We present a four-probe setup for measuring temperature of Joule-heated silicon in two independent ways from the same voltage measurement: a method using the thermal dependence of resistivity and a method based on the measured sheet power density. The two methods are compared to optical temperature measurements made by fitting a gray-body model onto data from a commercial spectrometer. The two four-probe temperature measurements are conducted from $890 \mathrm{~K}$ to $1540 \mathrm{~K}$, and they converge at temperatures above $1400 \mathrm{~K}$ indicating a high degree of self-consistency.
\end{abstract}

Published under license by AIP Publishing. https://doi.org/10.1063/5.0033465

\section{INTRODUCTION}

Measuring the temperature of semiconductors such as silicon during high temperature experiments is challenging. Yet it is important, for instance, for vapor phase epitaxy or chemical vapor deposition using microfabricated silicon heaters. ${ }^{1-3}$ Depending on measurement circumstances, temperature can be measured using a thermocouple drilled directly into a silicon sample, ${ }^{4,5}$ infrared spectroscopy, ${ }^{6,7}$ Raman spectroscopy, ${ }^{8}$ or a thermistor. ${ }^{2}$ In this paper, we present an alternative approach, where we explore two methods for obtaining the temperature of silicon from a single set of voltage measurements. Method A uses the silicon sample as a thermistor. Method B extracts the temperature from a sheet power density estimation of the sample. While the relation between temperature and resistivity in silicon has received extensive attention, only few attemps have been made to make a viable experimental method out of estimating temperature from a sheet power density measurement, ${ }^{13,14}$ and none of these have been applied to silicon.

The extracted temperature is compared with a set of optical measurements. A spectrometer is used to record a broad radiation spectrum alongside the electrical measurements. The temperature is obtained by a curve fitting of Planck's law to the spectrum as described in other studies. ${ }^{15,16}$

\section{THEORY}

When a current $I$ is passed from one end to the other of a rectangular slab as shown in Fig. 1, the sample temperature increases due to Joule heating. Using four-probe sensing, we measure the voltage drop between two contacts along opposite edges ( $V_{1}$ and $V_{2}$ in Fig. 1) of the slap. Two independent voltage measurements of the same segment serves to confirm reproducibility, and the temperature is now assessed in two separate ways. We shall assume the current density, and sample temperature $T$ is uniform.

\section{A. Method A-T $(\rho)$}

The sample resistivity $\rho$ is calculated from the measured voltage difference $V$ and sample dimensions,

$$
\rho=\frac{V}{I} \frac{w d}{L}
$$

where $L$ is the distance between the voltage contacts, $w$ is the width of the sample, and $d$ is the wafer thickness. The resistivity is related to the temperature by applying an experimentally fitted function. For intrinsic silicon, the relation between resistivity and temperature can 


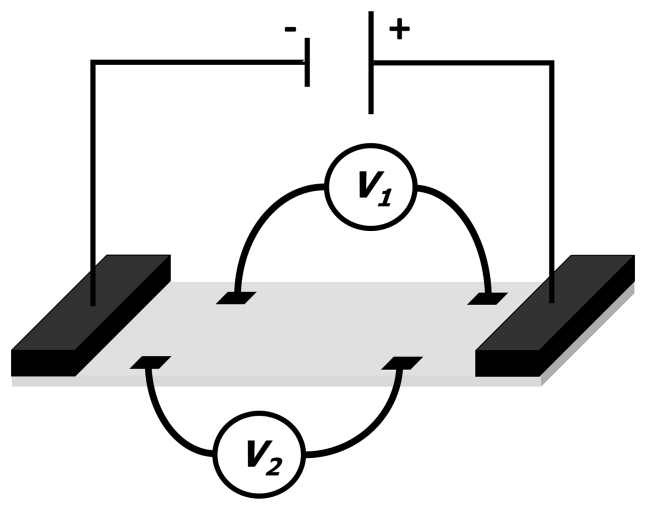

FIG. 1. Illustration of Joule heating of a slab with independent voltage measurements.

be approximated by ${ }^{4,12}$

$$
\begin{gathered}
T=\frac{\beta}{\log (\rho)-\alpha}, \\
T=\frac{\beta}{\log \left(\frac{V}{I} \frac{w d}{L}\right)-\alpha},
\end{gathered}
$$

with $\alpha=-4.2953$ and $\beta=2947.8$. The model has been extracted from data with a $1.8 \%$ error on the value of $\rho$ and an estimated $2 \mathrm{~K}$ error on the temperature measurements. ${ }^{4}$ We assume that there is no significant temperature variations along the wafer thickness, but note that we do measure an average of the bulk temperature.

\section{B. Method B-Radiative approximation}

The power dissipated as heat between the voltage probes is the product of the current and voltage. This heat must leave the segment by thermal conduction, convection, and radiation. At high temperatures, the heat lost through radiation greatly surpasses the heat lost through conduction (thermal convection is minimized by using a vacuum chamber). From the Stefan-Boltzmann law, the heat $Q$ radiated from a gray body depends on the temperature to the fourth power,

$$
Q=A_{S} \varepsilon \sigma\left(T^{4}-T_{0}^{4}\right),
$$

where $A_{S}$ is the surface area, $\sigma$ is the Stefan-Boltzmann constant, $T_{0}$ is the ambient temperature, and $\varepsilon$ is the emissivity of silicon (we used $\varepsilon=0.65^{17}$ ). Expressing the heat as the product of the current and voltage over the active area, the temperature becomes

$$
T=\sqrt[4]{\frac{I V}{2 w L \varepsilon \sigma}+T_{0}^{4}}
$$

We assume that the heat is radiated from the surface of the silicon sample, and thus, we measure the surface temperature with method B.

\section{Spectroscopy}

Radiation from heated silicon forms a spectrum. The intensity from a single spectral line is dependent on its wavelength, $\lambda$, and the temperature in accordance with Planck's law, ${ }^{18}$

$$
I_{\text {rad }}(\lambda, T)=\frac{P_{1}}{\lambda^{5}} \frac{1}{\mathrm{e}^{\frac{h c}{\lambda k_{\beta} T}}-1} .
$$

Here, $h$ is Planck's constant, $k_{\beta}$ is Boltzmann's constant, and $c$ is the speed of light. The temperature is found by fitting Eq. (6) to the measured intensity with variables $T$ and $P_{1}$.

\section{EXPERIMENTAL}

Experiments have been conducted using $800 \mu \mathrm{m}$ thick floatzone silicon samples of sizes between $10 \times 40 \mathrm{~mm}^{2}$ and $15 \times 50 \mathrm{~mm}^{2}$. The silicon used for the experiments was doped with phosphor and had a resistivity of $0.307 \Omega \mathrm{cm}-0.313 \Omega \mathrm{cm}$ at room temperature. To minimize contamination, oxidation, and convection cooling, samples were placed in a nitrogen-purged rough vacuum at $1.4 \times 10^{-2}$ Torr. Each sample was heated by passing a direct current (DC) from one end to the other along the length of the sample using molybdenum contacts. An Ocean Optics USB4000 spectrometer was used to record the intensity of the emitted spectrum from the center of the hot sample in the $200 \mathrm{~nm}-1100 \mathrm{~nm}$ range. Besides current leads on both ends, four molybdenum clamps were attached to the sample (see Fig. 2) allowing for pairwise independent measurements of the electrical potential between the probes at set currents using multimeters. Separate to the above-mentioned experiments, one test was carried out with an $\mathrm{N}$-type thermocouple tightly pressed to-but not drilled into-the center of a silicon sample.

\section{RESULTS}

The highest temperature recorded with the thermocouple was $1174 \mathrm{~K}$ at which point the sample melted. Thus, the thermocouple measurement resulted in a gross underestimation at high temperatures and was not used in subsequent experiments.

Three experiments were conducted with the combination of four-probe voltage measurements and spectroscopy data. We here present the result of one representative experiment. The voltage as a function of current is shown in Fig. 3. The acquired data from $V_{1}$ closely resemble the data from $V_{2}$, and the discrepancy is related to the distance between the voltage electrodes. This shows that the results are reproducible, and we can take the mean temperature evaluation of the two measurements to reduce the uncertainty.

The temperature of the silicon evaluated by methods $\mathrm{A}$ and $\mathrm{B}$ and spectroscopy is shown in Fig. 4. Method A was chosen as the reference because it showed a high consistency with both of the other methods over a large range. The temperature evaluated with method A and spectroscopy is in agreement between $890 \mathrm{~K}$ and $1340 \mathrm{~K}$, while method $\mathrm{B}$ is consistently higher in this temperature range. At temperatures above $1400 \mathrm{~K}$, methods A and B agree within the measurement uncertainty, while the spectroscopy deviates before completely failing. Fitting the spectroscopic data to the model proved ineffective above $1340 \mathrm{~K}$ as an unexpected signal appeared with a wavelength of $1 \mu \mathrm{m}$. With a different spectrometer of broader range calibrated to a blackbody, this issue can be avoided. 


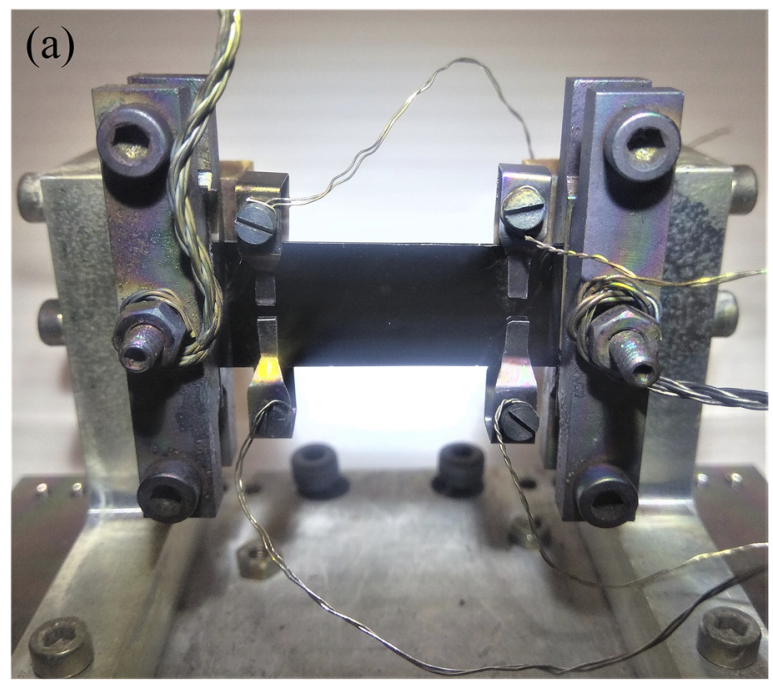

(b)

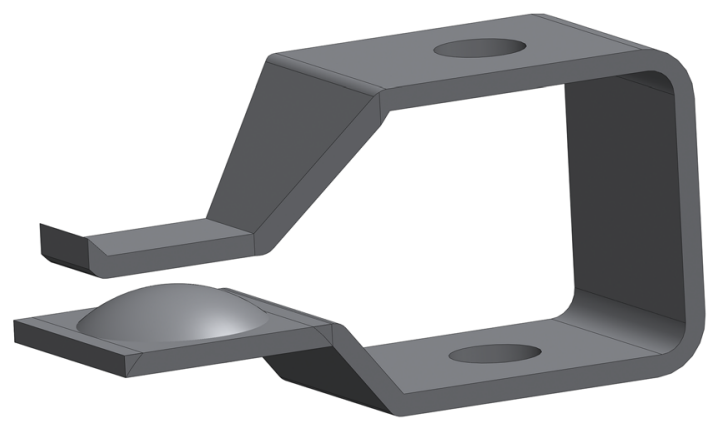

(c)

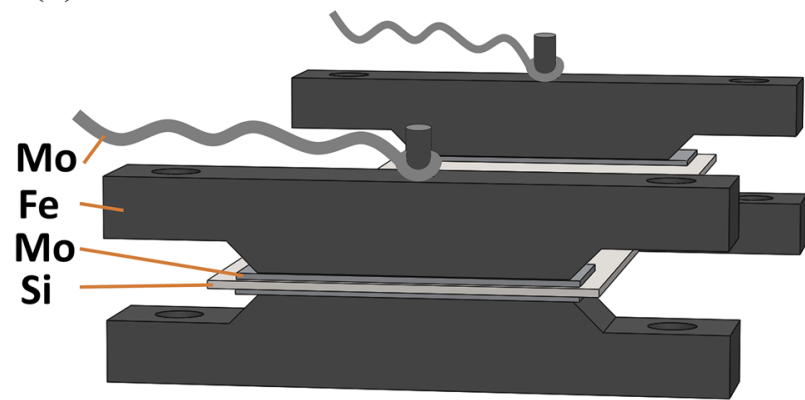

FIG. 2. (a) A silicon sample between two current leads mounted with four molybdenum clamps for voltage measurements, (b) the molybdenum clamp design, and (c) side view of the setup.

\section{DISCUSSION}

The requirements to the sample and the temperature range for which the methods can be assumed effective are not identical. This is true for the four-probes methods even though both originate from the same voltage measurement. Method A relies on an experimental

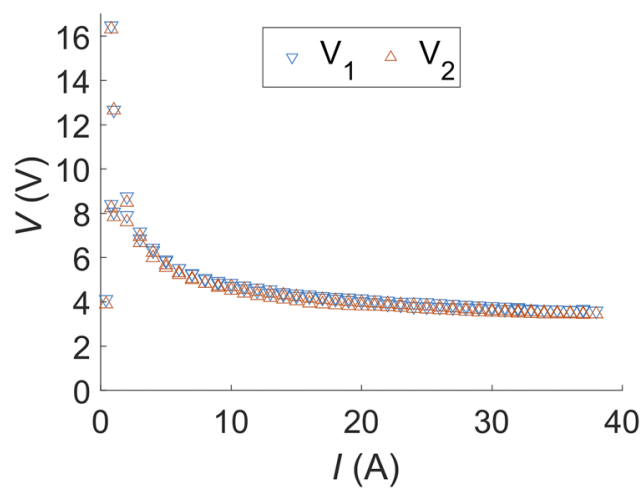

FIG. 3. Voltage measured as a function of DC current.

fit of the resistivity as a function of temperature for intrinsic silicon. Intrinsic silicon is challenging to heat with Joule heating as it is very resistive at room temperature. Low-doped silicon will however approach the behavior of intrinsic silicon at higher temperatures as the intrinsic carrier concentration surpasses the doping concentration (see Fig. 5), and the relative significance of impurity scattering on the resistivity with respect to lattice scattering diminishes. Only then can Eq. (2) be applied to the data.

The room temperature carrier concentration was $2 \times 10^{16} \mathrm{~cm}^{-3}$ for the silicon used in this study (calculation based on Ref. 20). At around $800 \mathrm{~K}$, the silicon behavior is qualitatively intrinsic with carrier concentration increasing with temperature; however, the intrinsic carriers only make up $58 \%$ of the total carriers at this point. This increases to more than $92 \%$ at $1000 \mathrm{~K}$ and from $1200 \mathrm{~K}$, more than $99 \%$ of the total carrier concentration stem from the intrinsic concentration.

Method B does not make requirements of the doping level of the sample. The calculated power equals the heat dissipated between the clamps at thermal equilibrium. However, the method relies on the assumption that the heat is dissipated through radiation from the surface and can only be expected to be accurate if all other forms of heat transfer are negligible in comparison. The most prominent

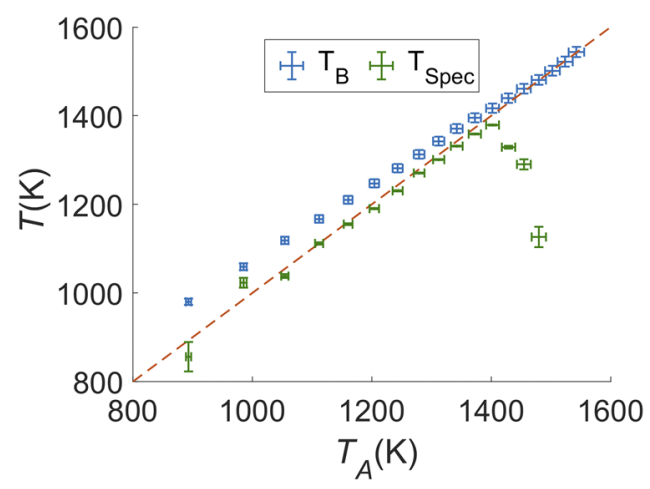

FIG. 4. Temperature as calculated by method $B$ and the spectroscopic curve fit as functions of the temperature as calculated by method A. Error bars represent the standard error of the mean for the electrical measurements and mark a $95 \%$ confidence interval on the spectroscopic curve fit. The red dashed line denotes a 1:1 relation, i.e., perfect correlation. 


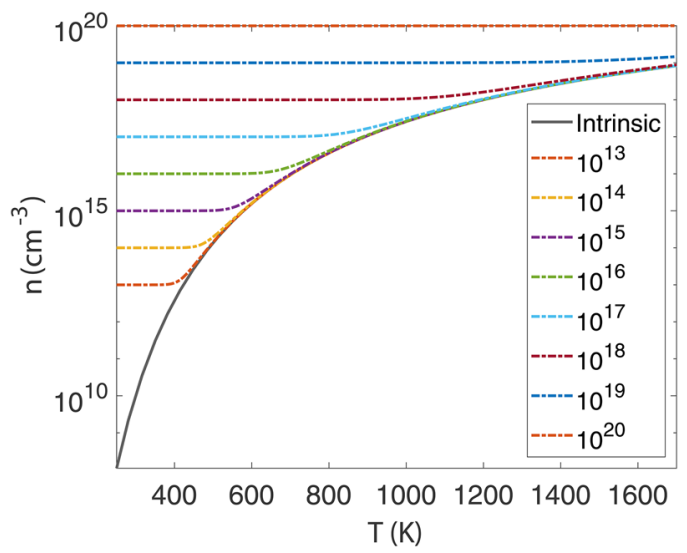

FIG. 5. A theoretical model for carrier concentration as a function of temperature (modeled from Ref. 19).

obstacle to achieve this is thermal conduction. As pointed out in other studies estimating temperature with the radiative approximation, ${ }^{13,14}$ the heat lost through thermal conduction cannot be determined generally. The amount of heat leaving the region between the clamps by conduction depends heavily on the specific setup, on the sample dimensions, and on how well the system is isolated. A rough model of the heat flux due to radiation and conduction is shown in Fig. 6. The model has been constructed in Matlab. It approximates the setup used in the experiment assuming a $300 \mathrm{~K}$ cold reservoir fixed at the ends of the $10 \mathrm{~cm}$ Mo wires constituting the leads to the sample. The center temperature of the sample is set at fixed temperatures up to $1681 \mathrm{~K}$. At each temperature, the conduction heat is calculated using the appropriate thermal conductivity for each material taking into account the temperature dependence of heat conductivity in silicon. ${ }^{21}$ This is put together with the calculated radiation heat from the silicon to give a good approximation of their relative sizes. Thermal radiation accounts for more than $99 \%$ of the heat loss at $1290 \mathrm{~K}$, and we take this as the lower bound for method B. An exact analytical solution to the problem is beyond the scope of this paper.

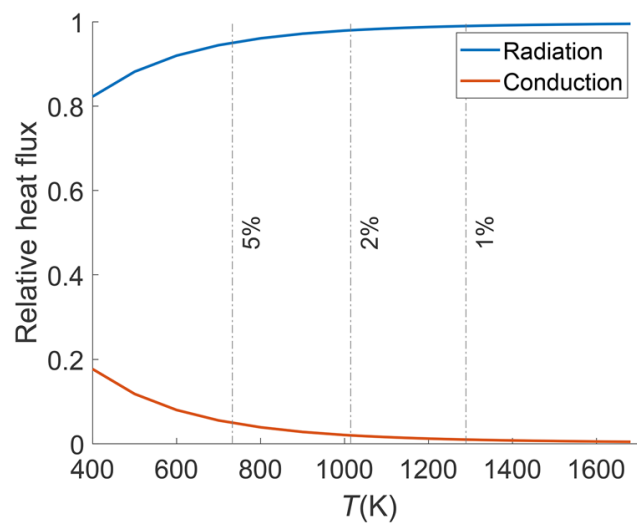

FIG. 6. Comparison of the heat flux due to radiation and heat flux due to conduction. $5 \%, 2 \%$, and $1 \%$ relative heat flux by conduction lies at $733 \mathrm{~K}, 1015 \mathrm{~K}$, and $1290 \mathrm{~K}$, respectively.
Commonly, both methods rely on the assumption that any thermoelectric effects are negligible. This is reasonable as the thermoelectric voltage is expected to be on the order of a few millivolt, ${ }^{4}$ while the measurements between the voltage electrodes are in the order of a few volt. It is also assumed that the sample is heated uniformly between the voltage probes. If the temperature varies along this length, method A will be based on an average resistivity, which cannot be related directly to an average temperature because the fitting function shows an inverse relation between $\log (\rho)$ and $T$. The same issue is not exhibited by method B.

The spectroscopic reading will depend on the amount of emitted light from the surface and generally requires the sample temperature to be at least a few hundred kelvin higher than room temperature to register a signal.

\section{A. Sensitivities}

The error on the actual electrical measurements used for both methods $\mathrm{A}$ and $\mathrm{B}$ is negligible in comparison to the error arising from uncertainties in the effective sample dimensions, i.e., the sample volume situated between the voltage probes. While the wafer producer provides specifications on the wafer thickness, the width and length of the area are measured with a caliper. Of these two measurements, the largest error originates from the uncertainty in the positioning of the voltage probes. This arises because the probe-sample contact area is of considerable size compared to the length of the area between the probes, thereby posing a resolution issue. We define the sensitivity with respect to the probe-probe distance, $L$, as

$$
S=\frac{\partial T / T}{\partial L / L}=\frac{\partial T}{\partial L} \frac{L}{T}
$$

This results in

$$
S_{A}=\frac{T}{\beta} .
$$

For method A, at $T=1200 \mathrm{~K}, S_{A} \approx 0.4$. The sensitivity of method B is

$$
S_{B}=-\frac{1}{4}
$$

with the condition $T \gg T_{0}$. Notice the sign change and similar magnitude. This means that an overestimation of $L$ will lead to an increase in temperature as calculated by method A but a decrease as calculated by method B, and thus, a sanity check to the methods can be performed.

\section{CONCLUSION}

We have demonstrated how four-probe sensing can be useful for determining the temperature of Joule-heated silicon at temperatures between $890 \mathrm{~K}$ and $1540 \mathrm{~K}$. Temperature measurements based on the thermal dependence of resistivity, method $\mathrm{A}$, and based on a sheet power density estimate, method $\mathrm{B}$, converge at temperatures higher than $1400 \mathrm{~K}$. This is important as they rely on distinct physical principles. The fact that they point to the same results in the highest of temperatures, where the degree of overestimation is minimal, demonstrates credibility. Their use as potential reference methods for optical temperature measurements has been shown by comparing electrically obtained temperature readings to analyze optical 
spectra from a commercial spectrometer. Albeit the electrical temperature measurements consistently surpass the spectroscopy estimates a little, there is a reasonable correlation between the two up to $1370 \mathrm{~K}$, and in fact, method A seems to be a more robust method for temperature estimation than the spectrometer in this study. As an added benefit to the electrical temperature assessment, the sensitivity of methods A and B shows reversed dependence on the probe-toprobe distance making it possible to conduct a sanity check of the setup near the melting point.

\section{ACKNOWLEDGMENTS}

This research was funded by the Innovation Fund Denmark (project TRIM). The authors are grateful for the support received from Topsil and would especially like to thank Anders Lei for his input and assistance. We would also like to express our gratitude to the DTU Photonics workshop and the DTU Physics workshop for a great job at manufacturing the experimental setup.

\section{DATA AVAILABILITY}

The data that support the findings of this study are available from the corresponding author upon reasonable request.

\section{REFERENCES}

'O. Englander, D. Christensen, and L. Lin, "Local synthesis of silicon nanowires and carbon nanotubes on microbridges," Appl. Phys. Lett. 82(26), 4797-4799 (2003).

${ }^{2}$ K. Mølhave, B. A. Wacaser, D. H. Petersen, J. B. Wagner, L. Samuelson, and P. Bøggild, "Epitaxial integration of nanowires in microsystems by local micrometer-scale vapor-phase epitaxy," Small 4(10), 1741-1746 (2008).

${ }^{3}$ D. S. Engstrøm, N. L. Rupesinghe, K. B. K. Teo, W. I. Milne, and P. Bøggild, "Vertically aligned CNT growth on a microfabricated silicon heater with integrated temperature control-Determination of the activation energy from a continuous thermal gradient," J. Micromech. Microeng. 21(1), 015004 (2010).

${ }^{4}$ W. Fulkerson, J. P. Moore, R. K. Williams, R. S. Graves, and D. L. McElroy, "Thermal conductivity, electrical resistivity, and Seebeck coefficient of silicon from 100 to $1300 \mathrm{~K}$," Phys. Rev. 167, 765-782 (1968).
${ }^{5}$ S. Fan, G. Plascencia, and T. Utigard, "High temperature electric conductivity of pure silicon," Can. Metall. Q. 47(4), 509-512 (2008).

${ }^{6} \mathrm{~T}$. Iuchi and T. Seo, "Temperature measurement of semitransparent silicon wafers based upon absorption edge wavelength shift," Int. J. Thermophys. 31(8), 1533-1543 (2010).

${ }^{7}$ J. C. Sturm, P. V. Schwartz, and P. M. Garone, "Silicon temperature measurement by infrared transmission for rapid thermal processing applications," Appl. Phys. Lett. 56(10), 961-963 (1990).

${ }^{8}$ M. R. Abel, T. L. Wright, W. P. King, and S. Graham, "Thermal metrology of silicon microstructures using Raman spectroscopy," IEEE Trans. Compon. Packag. Technol. 30(2), 200-208 (2007).

${ }^{9}$ E. H. Putley and W. H. Mitchell, "The electrical conductivity and Hall effect of silicon," Proc. Phys. Soc. 72(2), 193-200 (1958).

${ }^{10} \mathrm{~A}$. Hoffmann, K. Reuschel, and H. Rupprecht, "Measurement of the Hall effect and conductivity of super-pure silicon," J. Phys. Chem. Solids 11(3), 284-287 (1959).

${ }^{11}$ L. C. Burton and A. H. Madjid, "Coulomb screening in intrinsic mediumgap semiconductors and the electrical conductivity of silicon at elevated temperatures," Phys. Rev. 185, 1127-1132 (1969).

${ }^{12} \mathrm{M}$. A. Green, "Intrinsic concentration, effective densities of states, and effective mass in silicon," J. Appl. Phys. 67(6), 2944-2954 (1990).

${ }^{13} \mathrm{R}$. Raj, "Joule heating during flash-sintering," J. Eur. Ceram. Soc. 32(10), 2293-2301 (2012).

${ }^{14}$ R. I. Todd, E. Zapata-Solvas, R. S. Bonilla, T. Sneddon, and P. R. Wilshaw, "Electrical characteristics of flash sintering: Thermal runaway of Joule heating," J. Eur. Ceram. Soc. 35(6), 1865-1877 (2015).

${ }^{15}$ M. A. Khan, C. Allemand, and T. W. Eagar, "Noncontact temperature measurement. II. Least squares based techniques," Rev. Sci. Instrum. 62(2), 403-409 (1991).

${ }^{16} \mathrm{D}$. Ng and W. D. Williams, "Full-spectrum multiwavelength pyrometry for nongray surfaces," Proc. SPIE 1682, 260-270 (1992).

${ }^{17}$ F. G. Allen, "Emissivity at 0.65 micron of silicon and germanium at high temperatures," J. Appl. Phys. 28(12), 1510-1511 (1957).

${ }^{18}$ M. Planck, The Theory of Heat Radiation (P. Blakiston's Son \& Co, 1914).

${ }^{19} \mathrm{M}$. Glaubensklee, Analysis of the Temperature Dependence of Resistivity in N-Type Silicon (Defense Nuclear Agency, 1980).

${ }^{20} \mathrm{G}$. Masetti, M. Severi, and S. Solmi, "Modeling of carrier mobility against carrier concentration in arsenic-, phosphorus-, and boron-doped silicon," IEEE Trans. Electron Devices 30(7), 764-769 (1983).

${ }^{21}$ C. J. Glassbrenner and G. A. Slack, "Thermal conductivity of silicon and germanium from $3 \mathrm{~K}$ to the melting point," Phys. Rev. 134, A1058-A1069 (1964). 